\title{
Analyses de la dégradation du lac Kinkony pour la conservation du Complexe des Zones Humides Mahavavy-Kinkony, Région Boeny, Madagascar
}

\author{
Rado Andriamasimanana' and Mamy Rabarimanana" Correspondence: \\ Rado Andriamasimanana \\ Asity Madagascar, BP 1074 \\ Antananarivo 101, Madagascar \\ E-mail: rd.andriamasimanana @ birdlife-mada.org
}

\begin{abstract}
The Mahavavy-Kinkony wetland complex is an ecologically diverse and economically critical habitat assemblage. Despite recently receiving national protection, the ecological integrity of Lake Kinkony is threatened by the conversion of adjacent, unprotected lands into rice paddies. Conversion to aquacultural lands eliminates reed beds which provide favoured habitat for numerous endemic and endangered avian, fish and reptilian species, including Amaurornis olivieri, Paretroplus dambabe, P. kieneri and Erymnochelys madagascariensis. This research identified the physio-chemical sources and extent of degradation and evaluated the associated impacts on endangered wildlife. Employing digital environmental data within a Geographic Information System, the historical extent of reed habitat (circa 1949) was compared to distribution identified through fieldwork in 2008, indicating an areal loss of $80 \%$. Results indicate the primary contributor to reed loss was increased turbidity associated with erosion. The continuing decrease in marsh habitat facilitated by an increase in erosion poses significant threats to already endangered wildlife. However, while the reduction in marsh area was shown to reduce habitat availability for Amaurornis olivieri, observations imply this species does not occupy all potential reed habitats and that territorial preferences and tolerance towards turbidity need to be understood to accurately determine the magnitude of threat. Despite the need for continued research, information representing agents of change and their associated ramifications on fauna is essential for developing regional conservation and natural resource management strategies. In particular, anti-erosion management of the most vulnerable water catchment areas and restoration activities within the most severely degraded marshes are prescribed.
\end{abstract}

MOTS CLEFS : Kinkony, menaces, faune aquatique, turbidité, bassin versant.

KEYWORDS: Kinkony, threats, aquatic fauna, turbidity, watershed. 


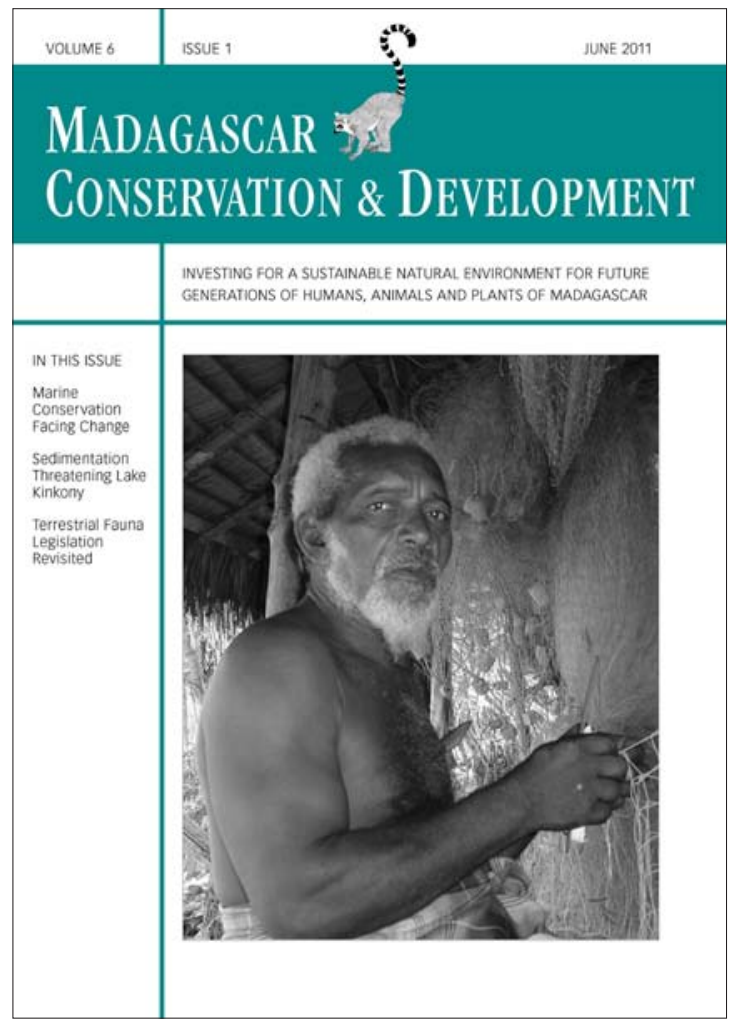

Madagascar Conservation \& Development is the journal of Indian Ocean e-Ink. It is produced under the responsibility of this institution. The views expressed in contributions to MCD are solely those of the authors and not those of the journal editors or the publisher.

All the Issues and articles are freely available at http://www.journalmcd.com

Contact Journal MCD

info@journalmcd.net for general inquiries regarding MCD funding@journalmcd.net to support the journal

Madagascar Conservation \& Development Institute and Museum of Anthropology University of Zurich

Winterthurerstrasse 190

CH-8057 Zurich, Switzerland

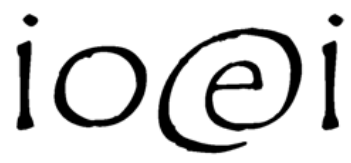

Indian Ocean e-Ink

Promoting African Publishing and Education www.ioeink.com

Missouri Botanical Garden (MBG)

Madagascar Research and Conservation Program BP 3391

Antananarivo, 101, Madagascar 


\section{INTRODUCTION}

Le lac Kinkony d'une superficie de l'ordre de 14000 ha est le deuxième plus grand lac de Madagascar après le lac Alaotra (Kiener 1963). Le lac Kinkony fait partie du Complexe des Zones Humides de Mahavavy-Kinkony qui est amené à devenir une Nouvelle Aire Protégée (NAP) de Madagascar (Office National pour l'Environnement 2009). La partie sud du lac Kinkony a été classée comme réserve de chasse selon l'arrêté n0126 SEHAEF / DIR/FOR du 13 janvier 1971 (Projet ZICOMA 1999). L'ensemble du Complexe profite d'un statut de protection temporaire suivant l'arrêté Interministériel n¹066/2006 MINENVEEF-MEM du 17 janvier 2007 et la gestion d'une superficie qui s'étend à l'est du lac a été récemment transférée aux communautés locales selon un arrêté communale n021/CR/ANT/09 signé en 2009. Le lac est depuis longtemps une des grandes zones de pêche des eaux intérieures de Madagascar (Kiener 1963) justifiée par la présence de poissons commerciaux dont dépendent plusieurs ménages riverains (Rafalimanana 1989, BioDev 2005) et qui constitue ainsi un enjeu économique important. Le lac Kinkony est soumis à des pressions anthropiques qui menacent la viabilité des ressources biologiques lacustres. Les menaces les plus importantes sont la conversion du pourtour du marais en rizières et la modification des propriétés de I'eau (Asity Madagascar 2009). Nous avons identifié les changements physico-chimiques au niveau du lac afin d'évaluer leur impact sur la faune menacée dont un oiseau, deux poissons et un reptile, respectivement la Marouette d'Olivier Amaurornis olivieri, Paretroplus dambabe et P. kieneri et la tortue Erymnochelys madagascariensis. L'analyse des changements environnementaux a été réalisée à partir d'outils d'investigation spatiale couplés à une analyse de la situation géographique du lac afin de cerner la problématique et interpréter les résultats obtenus sur l'importance écologique du lac Kinkony et de ses habitats limitrophes.

Le Complexe des Zones Humides Mahavavy-Kinkony est situé dans la région de Boeny, au nord-ouest de Madagascar dans le bassin du fleuve Mahavavy (Figure 1), entre les longitudes $\mathrm{E} 45^{\circ} 28^{\prime}$ et $45^{\circ} 56^{\prime}$ et les latitudes $\mathrm{S} 15^{\circ} 46^{\prime}$ et $16^{\circ} 12^{\prime}$, aux altitudes comprises entre le niveau de la mer et $150 \mathrm{~m}$ (Projet ZICOMA 1999). Situé dans la partie sud du Complexe, le lac Kinkony occupe à lui seul la moitié du plan d'eau continental du Complexe. La région du lac Kinkony est distribué dans l'étage sec, sous étage $2 b$ avec un déficit hydrique compris entre 400 et $700 \mathrm{~mm}$ et une moyenne des minimums du mois le plus froid compris entre 16 et $18^{\circ} \mathrm{C}$ conformément à la carte bioclimatique de Madagascar de Cornet (1974). La région présente une alternance avec une saison sèche marquée de sept mois d'avril à octobre et une saison pluvieuse de cinq mois, de novembre à mars. La moyenne des précipitations annuelles est d'environ 1550 mm, les mois les plus humides étant janvier et février (Projet ZICOMA 1999). La température moyenne annuelle est de $26^{\circ} \mathrm{C}$ avec un minimum de $18^{\circ} \mathrm{C}$ en juillet et un maximum de $35^{\circ} \mathrm{C}$ en décembre (Direction des Exploitations météorologiques 2003 In litt.).

Le lac Kinkony est entouré de petits lacs temporaires et permanents et ses parties sud et Sud-est sont alimentées par de nombreuses rivières temporaires et permanentes. II se déverse dans le canal de Kotomay, affluent de la Mahavavy. Un marécage de phragmites, qui constitue I'habitat naturel et spécifique de la Marouette d'Olivier Amaurornis olivieri frange sa bordure orientale (Figure 1). Une forêt sèche caducifoliée, la

\section{$45^{\circ} 30^{\prime} 0^{\prime \prime} \mathrm{E}$}

$45^{\circ} 40^{\prime} 0^{\prime \prime} \mathrm{E}$

$45^{\circ} 50^{\prime} 0^{\prime \prime} \mathrm{E}$

$46^{\circ} 0^{\prime} 0^{\prime \prime} \mathrm{E}$

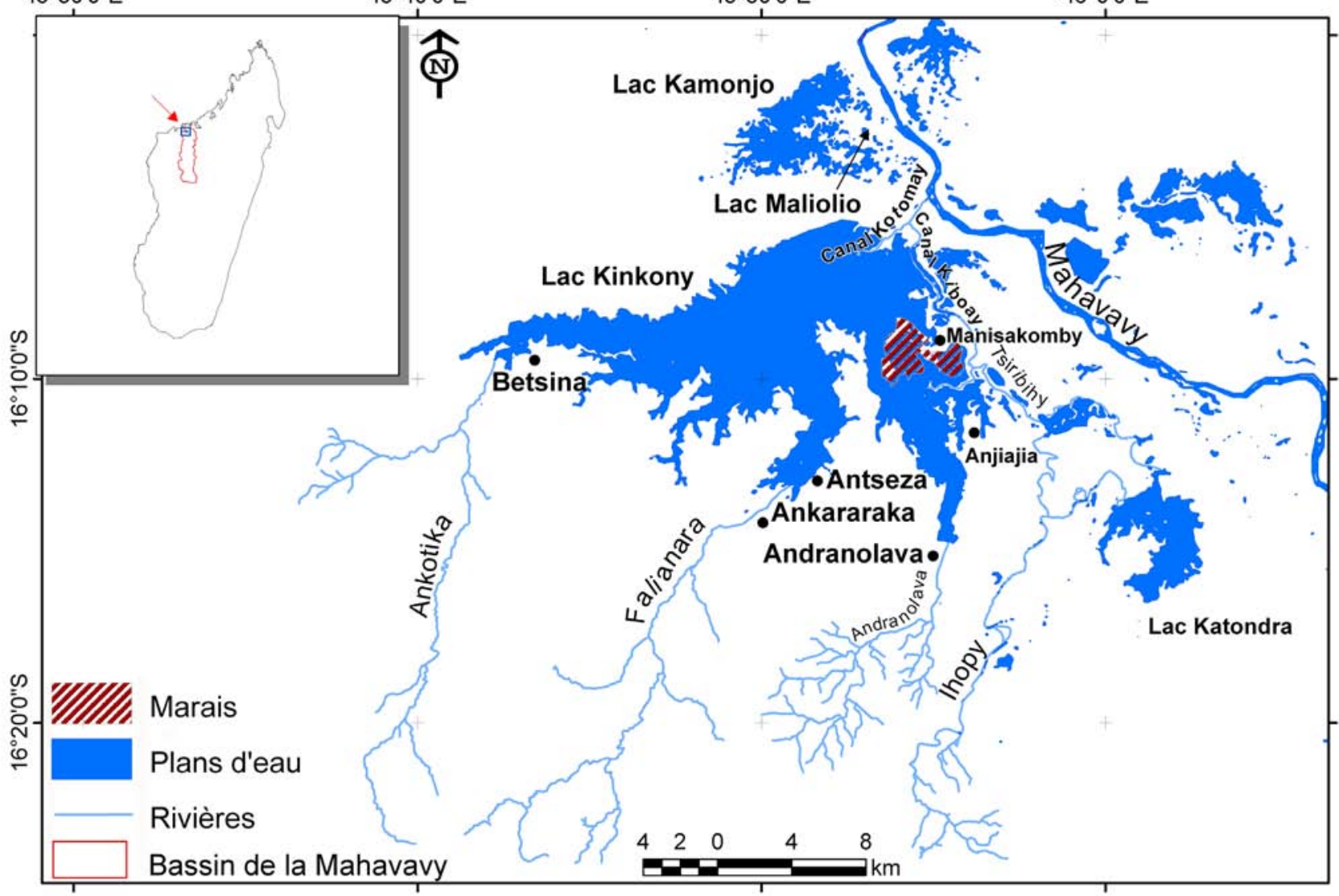

FIGURE 1. Localisation du marais et hydrographie du lac Kinkony. 
forêt de Marofandroaka, est située au nord du lac et se prolonge par une zone herbeuse à palmiers vers le nord-ouest. Une étude réalisée par BirdLife International (2004) a montré que le lac Kinkony est un lac dulçaquicole caractérisé par un $\mathrm{pH}$ variant entre 7,50 et 8,20. La température de l'eau à sa surface est de I'ordre de $25,5^{\circ} \mathrm{C}$ avec un taux d'oxygène variable compris entre 5,85 et $8,50 \mathrm{ml} / \mathrm{g}$ selon les endroits. La conductivité de l'eau se situe entre 0,26 et 0,35 millisiemens. Les turbidités mesurées près des villages de Manisakomby et d'Antseza sont respectivement de 32 et de $34 \mathrm{~cm}$. Le test portant sur les conditions physico-chimiques de l'eau du lac Kinkony a permis de conclure qu'elles sont favorables à la survie des espèces de poissons dulçaquicoles de Madagascar. Amaurornis olivieri est un oiseau endémique rare de la famille des Rallidae (Grandidier et Berlioz 1929) qui est extrêmement localisé (BirdLife International 2008) et classé comme espèce En Danger par I'Union Internationale pour la Conservation de la Nature (UICN) en 2010. L'évaluation effectuée par BirdLife International (2008) estime que l'espèce est représentée par moins d'un millier d'individus. Depuis son premier recensement en 1931 au lac Kinkony (Rand 1936), elle n'y a été redécouverte qu'en avril et mai 2003 (Anonyme 2003, Rabenandrasana et al. 2007). Les deux poissons Paretroplus dambabe (Sparks 2002) et $P$. kieneri (Arnoult 1960) de la famille des Cichlidae sont des espèces endémiques menacées (UICN 2010). P. dambabe est une espèce En Danger dont la distribution n'est actuellement connue que du Sud du delta de la Mahavavy en incluant le lac Kinkony et les lacs environnants (Loiselle 2004). P. kieneri est Vulnérable et sa distribution englobe le sud du bassin de la Mahavavy ainsi que le Nord des bassins de la Betsiboka et de la Mahajamba (Kiener 1963, Loiselle et al. 2004). La tortue Erymnochelys madagascariensis (Grandidier 1867) est endémique de Madagascar et appartient à la famille des Podocnemidae. II s'agit d'une tortue aquatique qui fréquente les eaux douces (Glaw et Vences 2007) et qui est classée En Danger Critique (UICN 2010). Elle est principalement herbivore et occasionnellement carnivore, en se nourrissant principalement de plantes aquatiques (Henkel et Schmid 2000). Elle est localisée dans les zones de basse altitude du versant Ouest de l'île entre le fleuve Mangoky au sud et la région du Sambirano (Glaw et Vences 2007).

Dans le cadre de la gestion du Système des Aires Protégées de Madagascar, les NAP sont proposées avec une stratégie de développement durable dans laquelle l'écotourisme constitue I'un des axes principaux. Les visites ornithologiques pour découvrir l'espèce phare, la Marouette d'Olivier, et d'autres oiseaux d'eau sont les atouts du site (Asity Madagascar 2009). Il est donc important de maintenir la viabilité de ces espèces pour assurer le développement durable de la région, de sorte que l'évaluation des menaces qui pèsent sur les habitats de ces espèces menacées s'avère être la recherche prioritaire à mener pour le site.

\section{MATÉRIELS ET MÉTHODOLOGIE}

La méthodologie adoptée fait appel à des outils d'investigation et de modélisation spatiale comme la Télédétection et le Système d'Information Géographique (SIG) pour analyser les changements au niveau des habitats naturels du lac. Les données de base sont les images satellitaires Landsat (1973, 1989, 1995, 1999 et 2005), la carte pédologique (Ségalen 1952), la carte topographique du Foiben-Taosaritanin'i Madagasikara
(FTM 1956), le modèle numérique de terrain créé à partir de la carte FTM (1956). Pour mesurer la turbidité de l'eau, nous avons utilisé le disque secchi et pour délimiter les zones de phragmites, nous avons utilisé un Global Positionning System (GPS) couplé à un ordinateur équipé de logiciels de traitement d'images (Idrisi Andes de Clark labs) et de cartographie (ArcGIS d'ESRI).

La carte topographique du FTM (1956), établie à partir de photographies aériennes prises en juin 1949, a été utilisée comme référence pour évaluer la réduction de la superficie des phragmites entre 1949 et nos recensements sur le terrain en avril 2008. Dans la mesure où la superficie en eau et l'étendue du marécage du lac Kinkony varient avec les saisons, nous avons calé nos missions de terrain en 2008 pour nous conformer à la saison des ortho - photographies de juin 1949 afin d'évaluer la variation de la superficie de la phragmitaie entre 1949 et 2008. D'autres types de données dont le réseau hydrographique (FTM 1949) et le modèle numérique de terrain (MNT) utilisés pour délimiter les bassins ont été extraits de cette carte topographique ont servi de référence au cours de la recherche portant sur l'érosion. Les données ci-dessus ont été complétées par une série d'images satellite Landsat à 30m de résolution, dont Landsat MMS de juillet 1973, Landsat TM d'avril 1989, de juin 1994 et d'avril 2005 et Landsat ETM+ de septembre 1999. Deux missions de terrain ont été effectuées, dont une mission d'une semaine en avril 2008 pour identifier l'étendue de la phragmitaie avec un GPS et une mission d'une semaine en mars 2010 pour relever les données sur la turbidité du lac. Pour cartographier les changements, nous avons procédé à une analyse spatiale en numérisant la phragmitaie identifiée sur la carte de 1956 pour servir de référence à l'étendue de l'habitat d'Amaurornis olivieri pour l'année 1949. Les points obtenus avec le GPS en 2008 ont été convertis dans une projection identique à celle utilisée en 1949 afin de comparer les superficies de la phragmitaie entre 1949 et 2008 avec les outils du SIG.

L'identification des zones de vulnérabilité à l'érosion a été réalisée à partir des éléments numérisés des données topographiques de 1949. Les facteurs d'érosion ont été calculés selon la formule empirique de Wischmeier (1960), soit :

$$
A=R * K * L S * C * P,
$$

avec $A$ = perte de sol par érosion exprimée en tonnes / ha / an ; $\mathrm{R}=$ index d'érosivité des pluies ; $\mathrm{K}$ = indice d'érodabilité du sol ; $\mathrm{LS}=$ indice topographique dont $\mathrm{L}$ est la longueur de la pente en $\mathrm{m}$ et $\mathrm{S}$ est I'inclinaison de la pente en pourcent (\%) ; $\mathrm{C}=$ indice de couverture végétale; et $\mathrm{P}=$ indice de traitement antiérosif. Ces facteurs ont été traités dans le programme d'analyses spatiales de I'outil ModelBuilder ${ }^{\top M}$ de l'extension d'ArcView Spatial Analyst (ESRI) pour identifier les bassins les plus vulnérables à l'érosion en identifiant les pixels les plus vulnérables et les secteurs qui requerraient des aménagements urgents (Morschel et Fox 2004). L'index d'érosivité des pluies R n'a pas été considéré car nous avons admis que l'intensité des pluies était la même sur tout le pourtour du lac Kinkony, nous avons ainsi considéré une valeur de 1,204 pour tous les bassins, à savoir la valeur obtenue dans les parcelles élémentaires de la zone occidentale (Bailly et al. 1976). L'index d'érodabilité du sol K a été modélisé à partir de la carte pédologique de Ségalen (1952) et des classes identifiées (Tableau 1). L'érodabilité diminue du sol latéritique aux sédiments de dépôts alluvionnaires, respectivement connus sous leur terminologie de "ferrasol, oxisol» 
et «fluvisol, entisol» (World Reference Base for Soil Resources et Soil Taxonomy 1998). L'indice topographique LS englobe le degré et la longueur de la pente, plus la pente est forte et longue plus l'érosion est forte. Le calcul de la pente a été réalisé avec MNT pour chaque bassin. L'indice de la couverture végétale $C$ varie entre les valeurs 0 et 1 ; plus le sol est couvert de végétation, plus sa valeur diminue et moins le sol est érodable, un sol nu ayant un indice de 1 (Roose 1977). L'indice C est basé sur I'indice de végétation obtenu du modèle NDVI (Normalized Difference Vegetation Index) et I'indice de brillance. Ces indices ont été calculés à partir de l'image satellite la plus récente de la zone, celle de 2005. Dans l'étape suivante, tous ces facteurs ont été répartis en 10 classes numérotés de 0 à 9 pour décrire la contribution à l'érosion du minimum (0) au maximum (9) avant de les intégrer à la carte pour calculer la valeur de la vulnérabilité du sol à l'érosion, puis de la vulnérabilité par bassin versant. Au terme de la modélisation, chaque unité de l'image résultante, qui est représentée par un pixel, possède une valeur qui indique sa vulnérabilité à l'érosion. Étant donné que l'objectif est d'évaluer la vulnérabilité au niveau de chaque bassin, l'ensemble de la zone d'étude a été découpé par bassin identifié et les valeurs des pixels ont été sommées au niveau de chaque bassin. Ces totaux par bassin ont été divisés par la somme de tous les bassins considérés sur le site pour pouvoir comparer entre elles les valeurs de la vulnérabilité des bassins.

TABLEAU 1. Mode de classification des types pédologiques d'après leur érodabilité.

\begin{tabular}{|c|c|c|}
\hline Description & Érodabilité & Mode de classification \\
\hline Alluvion argileux & Moins & $\begin{array}{l}\text { pente }=0 \\
\text { (zone de sédimentation) }\end{array}$ \\
\hline Alluvion argilo-limoneux & & $\begin{array}{l}\text { pente }=0 \\
\text { (zone de sédimentation) }\end{array}$ \\
\hline $\begin{array}{l}\text { Alluvion calcaire non } \\
\text { micacé }\end{array}$ & & $\begin{array}{l}\text { pente }=0 \\
\text { (zone de sédimentation) }\end{array}$ \\
\hline Alluvion mica limoneux & & $\begin{array}{l}\text { pente }=0 \\
\text { (zone de sédimentation) }\end{array}$ \\
\hline Sol tany manga & & ancien lit (ancien dépôt) \\
\hline Sol tacheté argileux & & ancien lit (ancien dépôt) \\
\hline Sol hydromorphe lessive & & ancien lit (ancien dépôt) \\
\hline $\begin{array}{l}\text { Basalte (sol d'érosion ou } \\
\text { squelettique) }\end{array}$ & & massif \\
\hline $\begin{array}{l}\text { Calcaire (sol d'érosion ou } \\
\text { squelettique) }\end{array}$ & & massif \\
\hline $\begin{array}{l}\text { Marne (sol d'érosion ou } \\
\text { squelettique) }\end{array}$ & & massif résiduel \\
\hline $\begin{array}{l}\text { Meulière (sol d'érosion ou } \\
\text { squelettique) }\end{array}$ & & massif résiduel \\
\hline $\begin{array}{l}\text { Grès (sol d'érosion ou } \\
\text { squelettique) }\end{array}$ & & massif résiduel \\
\hline Collusion (sol d'apport) & & dépôt consolidé \\
\hline Sol ferrugineux Basalte & & altérite basique \\
\hline Sol jaune basalte & & altérite basique \\
\hline Sol ferrugineux calcaire & & altérite calcique \\
\hline Sol latéritique calcaire & & altérite calcique \\
\hline Sol jaune gréseux & & altérite siliceux \\
\hline Sol jaune sableux & & altérite siliceux \\
\hline Sol rouge gréseux & & altérite siliceux \\
\hline Sol rouge sableux & Plus & altérite siliceux \\
\hline
\end{tabular}

Le contrôle sur le terrain a été réalisé en mars 2010 au cours duquel des mesures de turbidité et de la profondeur du lac ont été effectuées en utilisant un disque de Secchi. Ces mesures ont été faites en saison de pluies, ce qui permet de supposer que les rivières temporaires et permanentes des bassins contribuaient de façon égale à la turbidité. Pour chaque point d'échantillonnage, la valeur notée est la dernière indiquée par le disque de Secchi avant que celui-ci ne devienne invisible dans l'eau. Ainsi, plus la valeur est petite, plus l'eau est trouble et inversement. Ce contrôle a servi à évaluer le modèle d'érosion élaboré à travers une analyse de corrélation spatiale en comparant les valeurs de vulnérabilité avec celles de la turbidité relevées au niveau des sources des divers cours d'eau des bassins versants. Une interpolation des valeurs sur l'ensemble du lac a été réalisée pour établir la concordance avec I'analyse de l'érosion des bassins. Les eaux de ruissellement sont l'agent de transport des particules et des sédiments dans les lacs. En suspension dans I'eau, ces particules empêchent l'entrée de la lumière dans l'eau et conduisent à la diminution de la photosynthèse effectuée par les plantes aquatiques. Cette situation conduit à une diminution du taux d'oxygène dissous dans l'eau, phénomène qui pourrait affecter la biologie de la faune aquatique. Dans des conditions extrêmes elle engendre la mort des poissons (Francis-Floyd 1992).

\section{RÉSULTATS}

Les images satellite disponibles montrent que la superficie du lac pouvait atteindre 15543 ha à la fin de la saison des pluies (avril 2005) et se réduire à 12062 ha à la fin de la saison sèche (septembre 1999), soit une réduction de plus de $22 \%$. Les mesures sur le terrain et leur traitement informatique ont permis de déterminer que les phragmitaies du lac Kinkony occupaient une superficie de 71 ha en septembre 2008 contre 540 ha évalués d'après la carte topographique de 1949 (Figure 2). Nos estimations sur le terrain nous ont permis d'évaluer que la zone marécageuse est constituée par $80 \%$ de phragmites et $20 \%$ d'autres végétations flottantes. Le MNT a permis de délimiter les bassins versants du pourtour du lac Kinkony qui sont d'une superficie totale de 63000 ha. Quatre bassins sont plus particulièrement vulnérables à l'érosion avec l'indice de vulnérabilité le plus élevé $(0,220)$ sur le bassin de la rivière Ihopy qui alimente le lac Katondra, qui lui se déverse dans la rivière Tsiribihy qui coule entre les localités d'Anjiajia et Manisakomby (communément appelé Makary) et se prolonge par le canal de Kiboay au nord de Manisakomby qui alimente le lac Kinkony. Le second bassin est celui de la rivière Ankotika et du ruisseau Kabosy avec ses affluents qui alimentent le lac au niveau de Betsina avec un indice de 0,132. Le troisième bassin est celui des rivières Ankararaka et Falianara et de leurs affluents qui alimentent le lac au niveau d'Antseza où l'indice était de 0,089 et le quatrième bassin est celui du ruisseau d'Andranolava qui alimente le lac au niveau du village d'Andranolava où un indice de 0,048 a été relevé (Figure 1).

La mesure de la turbidité a été réalisée en 20 points avec des valeurs de transparence variant de $28 \mathrm{~cm}$ à $148 \mathrm{~cm}$. Quatre localités présentent une forte turbidité de l'eau dont I'embouchure du lac à l'extrémité ouest (Betsina), une localité près d'Antseza, I'extrémité sud du lac Kinkony et une localité à l'est du lac au niveau des canaux Kotomay et Kiboay au nord de Makary (Figures 1, 2). 


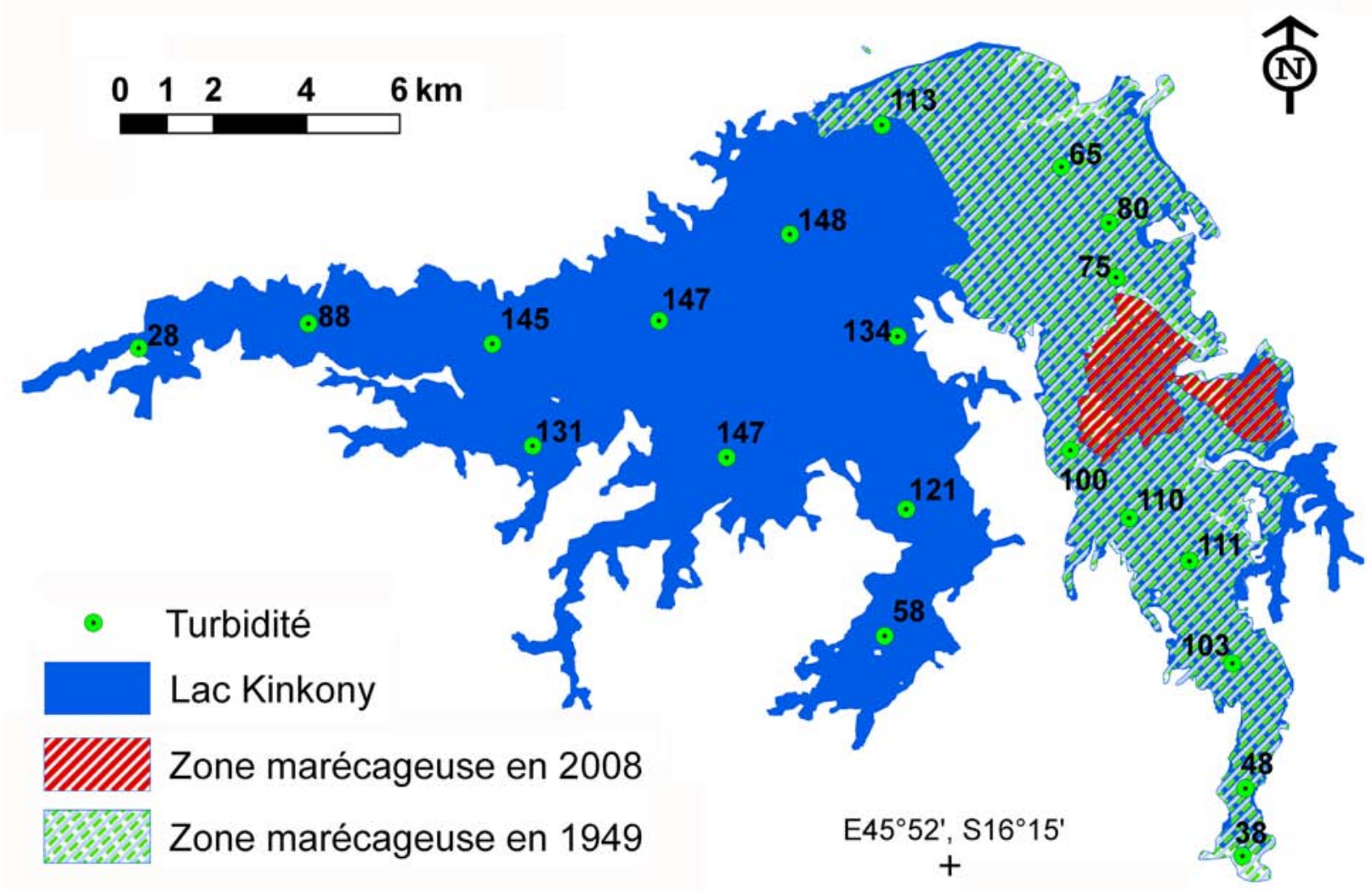

FIGURE 2. Étendues historique et actuelle des zones marécageuses du lac Kinkony et indices de turbidité relevés en 2008.

La mesure de la profondeur a montré des valeurs variant de 2,15 à 8,21 m. La droite de régression établie pour la turbidité et la profondeur a révélé une forte corrélation positive entre ces deux variables conformément au schéma classique montré par Fowler et Cohen (1986). Cette corrélation est exprimée dans le cas du lac Kinkony par l'équation profondeur $=65,234$ $+4,402 *$ turbidité avec le coefficient de corrélation $r=0,872$. Le test de Student a montré une valeur de $t=7,565$, très supérieure à celle de la table de distribution avec une probabilité 0,01 . Cela signifie que la corrélation linéaire entre la turbidité et la profondeur est significative.

\section{DISCUSSION}

Les séries d'images satellite Landsat prises entre 1973 et 2005 ont permis de montrer les variations saisonnières importantes de la superficie du lac. Les cartes numérisées obtenues à partir des traitements d'images n'ont pas permis de distinguer les phragmitaies des zones herbeuses, en même temps que les aspects saisonniers biaisaient l'ensemble de l'interprétation. Les enquêtes auprès des communautés locales nous ont permis d'apprendre que lors des plus grandes crues, plus particulièrement lors du passage de cyclones, la superficie du lac Kinkony pouvait s'étendre largement en provoquant un important éclaircissement des phragmitaies. Au cours de tels épisodes, il est difficile d'évaluer la superficie des phragmites sur le terrain comme à partir d'images satellite ou de photos aériennes mais aucune des images que nous avons utilisée ne correspond à de tels événements cycloniques. Nous avons résolu ce problème en procédant à une mesure directe de la surface des phragmites au cours d'une mission de contrôle sur le terrain en avril 2008. Nous avons estimé que ces estimations de superficie sont fiables pour ce contexte dans la mesure où les photographies aériennes étaient de haute résolution, de I'ordre du $\mathrm{cm}$, et que les erreurs de positionnement par GPS étaient de l'ordre de $20 \mathrm{~m}$.

La carte topographique établie en 1949 illustre un marais sur la partie orientale du lac Kinkony sans donner aucune autre précision. Si nous pouvons considérer qu'il s'agissait en grande partie d'une phragmitaie, dans les proportions de $80 \%-20 \%$ observées aujourd'hui, celle-ci aurait vu une réduction de sa superficie de $80 \%$ en 59 ans. Cette diminution a forcément affecté Amaurornis olivieri ainsi que les deux espèces de poissons Paretroplus dambabe et $P$. kieneri car ces espèces dépendent de ce milieu pour la reproduction.

Au cours de notre visite sur le terrain, nous avons constaté qu'Amaurornis olivieri n'occupait pas toutes les phragmitaies du lac Kinkony en étant absente d'une importante portion du lac. Les guides locaux avaient une connaissance fine du territoire occupée par cette espèce et en faisaient profiter les chercheurs sur le site. La densité de la population d'Amaurornis olivieri au niveau des phragmitaies du lac a été estimée à 7,6 individus par $\mathrm{km}^{2}$ (Rabenadrasana et al. 2009). II est vraisemblable que des facteurs écologiques liés à sa nidification pourraient limiter I'étendue de sa distribution mais des études sont requises pour mieux connaître Amaurornis olivieri, d'autant que rien ne nous a permis de comprendre pourquoi une phragmitaie d'assez grande superficie au sud, appelée marais d'Anjanaborona, n'était pas occupée par l'espèce. La phragmitaie où se trouve actuellement I'oiseau et celle d'Anjanaborona se trouvent sur le même plan d'eau et ne sont distantes que de $70 \mathrm{~m}$. Les deux poissons endémiques Paretroplus dambabe et $P$. kieneri sont présents dans le grand lac Kinkony mais si $P$. dambabe 
est inféodé au lac Kinkony, P. kieneri est également rencontré dans le lac Maliolio qui se trouve à l'est du lac Kamonjo. II a été proposé que les types de végétation flottante du lac Kinkony joueraient un rôle important en fournissant une zone de fraie pour ces poissons (BirdLife International Madagascar Programme 2006) et en affectant ainsi leur reproduction. Dans la mesure où $P$. dambabe n'est distribué que sur le grand lac, il serait plus vulnérable à cette menace que $P$. kieneri.

En combinant les modélisations de la turbidité et de la vulnérabilité des bassins à l'érosion, on voit nettement les relations entre ces deux caractéristiques. Les quatre bassins versants classés les plus vulnérables à l'érosion ont leur exutoire dans les portions du lac où nous avons relevé de fortes valeurs de turbidité. Ces bassins semblent être les principaux contributeurs à la turbidité du lac Kinkony dans la mesure où les modèles de la turbidité sont conformes à ceux de la vulnérabilité à l'érosion des bassins.

Des études plus avancées devraient être menées pour appréhender l'influence de la turbidité sur le taux d'oxygène dissous dans l'eau. Dans la mesure où le lac a un exutoire vers le fleuve Mahavavy, surtout en période de décrues, des variations de turbidité du lac devraient exister au cours des différentes saisons de I'année, variations qui devraient être documentées par des mesures régulières. Les plans d'eau avec des valeurs de turbidité constamment élevées tout au Iong de l'année constitueraient des zones de menace pour la survie de la faune aquatique.

La forte corrélation entre la turbidité et la profondeur du lac montre que plus la turbidité est élevée, moins l'eau est profonde. Cela montre que le comblement du lac au cours des temps est dû aux dépôts de sédiments. En effet, un programme de suivi de la variation de la profondeur de I'eau devrait être mis en place pour l'évaluation à long terme de sont état de comblement. L'effet combiné du comblement et du changement climatique devrait également être analysé car le risque d'assèchement définitif du lac pourrait s'accentuer. L'installation d'une échelle limnimétrique s'avère donc indispensable afin de suivre la variation de la hauteur d'eau annuelle.

L'importance des menaces sur Erymnochelys madagascariensis est difficile à estimer. Aucune information ne nous permet d'estimer si la diminution de la végétation flottante ou l'érosion affectent directement la biologie de cette espèce de tortue aquatique. Néanmoins, I'érosion qui provoque I'envasement du lac va favoriser la conversion du plan d'eau vers d'autres modes d'occupation telles que les rizières et le marécage (Conservation Breeding Specialist Group 2002).

La riziculture pratiquée par la population riveraine du lac est particulière car elle suit la décrue des eaux sur les rives. L'envasement par une érosion modérée favorise donc cette pratique locale, plus particulièrement sur les bords peu profonds. L'expansion de la riziculture est surtout visible sur la partie occidentale du lac Kinkony, près du village de Betsina, où le plan d'eau recule considérablement chaque année pour laisser la rizière avancer. Au niveau des embouchures près de Bestina, d'Antseza et d'Andranolava, les deux espèces de poissons (Paretroplus dambabe et $P$. kieneri) ainsi que la tortue (Erymnochelys madagascariensis) sont menacées par l'action combinée de la réduction de l'espace vital et la forte turbidité.

\section{CONCLUSION}

Les analyses de la corrélation spatiale entre la turbidité mesurée et la vulnérabilité des bassins versants à l'érosion indiquent que l'érosion est une des sources de la dégradation physico-chimique du lac Kinkony. La présente étude a également permis de localiser les zones vulnérables où d'importants volumes de sédiments sont déposés dans l'eau du lac. En l'état de nos connaissances, nous n'avons pas identifié le seuil audelà duquel la turbidité affecte réellement la biologie de la faune aquatique du lac. Une étude approfondie des espèces devrait être menée pour identifier les effets de l'érosion sur la distribution de l'oxygène dissous dans l'eau ainsi que celle de la faune menacée du lac. Des enquêtes sur les impacts du changement climatique devraient être menées afin de formuler les mesures d'adaptation locales et adéquates pour adopter les mesures pertinentes dans la préservation de ces ressources lacustres. La protection des lacs est souvent plus complexe que celle d'autres écosystèmes dont la préservation peut s'entendre localement car dans les milieux lacustres, il faut considérer la protection à une échelle globale en tenant compte des bassins versants en amont dans toutes les considérations et veiller à ce que les aménagements nécessaires soient prévus dans le Plan d'Aménagement et de Gestion. Malgré les incertitudes sur le niveau de turbidité, la stratégie de conservation du site doit inclure des plans d'aménagement pour lutter contre l'érosion dans les quatre bassins versants identifiés comme étant les plus vulnérables et envisager des activités de restauration des phragmitaies.

\section{REMERCIEMENTS}

Nous voulons adresser nos vifs remerciements aux organisations et personnes qui nous ont aidé à la réalisation de cette recherche ; International Foundation for Sciences, Conservation International ainsi que Idea Wild pour les appuis financiers et matériels ; BirdLife International et Asity Madagascar plus particulièrement pour l'appui logistique, les ressources humaines et pour l'accès aux données et aux sites ; toutes les personnes qui nous ont aidé de près ou de loin dans l'exécution de cette recherche, et plus particulièrement Holisoa Andriamanantena, Voninavoko Raminoarisoa, Alison Cameron, Rivo Rabarisoa, Roger Safford, ainsi que les communautés locales du MahavavyKinkony. Nous tenons également à remercier les éditeurs du journal et trois rapporteurs anonymes qui ont grandement amélioré cet article.

\section{BIBLIOGRAPHIE}

Anonyme 2003. Recent reports. Madagascar. Bulletin of the African Bird Club 10: 135-136.

Asity Madagascar 2009. Plan d'Aménagement et de Gestion du Complexe Zones Humides Mahavavy-Kinkony, Région Boeny. Rapport non publié.

BioDev 2005. Analyse Socio-Économique de la Mise en Place d'un Site de Conservation dans le Complexe Mahavavy-Kinkony. Rapport non publié remis à BirdLife International Madagascar Programme.

BirdLife International Madagascar Program 2004. Évaluation Écologique et Identification des Sites Potentiels pour la Conservation de la Biodiversité des Zones Humides du Complexe Mahavavy-Kinkony (Province de Majunga). Rapport non publié.

BirdLife International Madagascar Program 2006.Schémas d'Aménagement du Complexe Zones Humides Mahavavy-Kinkony, Région Boeny. Rapport non publié.

BirdLife 2008. Threatened birds of the world 2008. CD-ROM 
Chaperon, P., Danloux, J. et Ferry, L. 2005. Fleuves et Rivières de Madagascar. ORSTOM, Paris.IRD

Conservation Breeding Specialist Group 2002. Évaluation et Plans de Gestion pour la Conservation (CAMP) de la Faune de Madagascar: Lémuriens, autres Mammifères, Reptiles et Amphibiens, Poissons d'eau douce et Évaluation de la Viabilité des Populations et des Habitats de Hypogeomys antimena (Vositse). IUCN Species Survival Commission. <http://www.biodiv.be/madagascar/implementation/ Documents_nationaux/madagascar-camp> téléchargé le 23 avril2010

Cornet, A. 1974. Essai de cartographie bioclimatique à Madagascar. Notice explicative $n^{\circ} 55$. ORSTOM, Paris.

Deckers, J., Nachtergaele, F. \& Spaargaren, O. 2003. Tropical Soils in the Classification Systems of USDA, FAO and WRB. <http://www.fao.org/ag/ agll/wrb/doc/KAOWDeckerscorr280203.doc> téléchargé le 20 avril 2010

Fowler, J. \& Cohen, L. 1986. Statistics for ornithologists. Second edition. British Trust of Ornithology, Tring.

Francis-Floyd, R. 1992. Dissolved Oxygen for Fish Production. Fact Sheet FA 27, Department of Fisheries and Aquaculture, Florida Cooperative Extension Service, Institute of Food and Agricultural Sciences, University of Florida.

Friedrich-Wilhelm, H. \& Schmidt, W. 2000. Amphibians and Reptiles of Madagascar and Mascarene, Seychelles, and Comoro Islands. Krieger Publishing, Malabar, Florida.

Glaw, F. \& Vences, M. 2007. A Field Guide to the Amphibians and Reptiles of Madagascar. Third Edition. Vences \& Glaw Verlag, Köln, Germany.

Guillaumet, J.-L. et Koechlin, J. 1971. Contribution à la définition des types de végétation dans les régions tropicales (exemple de Madagascar). Candollea 26, 2: 263-277.

IUCN 2010. IUCN Red List of Threatened Species. Version 2010.1. <WWW. iucnredlist.org> téléchargé le 19 avril 2010

Kiener, A. 1963. Poissons, Pêche et Pisciculture à Madagascar. Publication 24. Centre Technique Forestier Tropical, Nogent sur Marne, France.

Loiselle, P. 2004. Paretroplus dambabe. In IUCN 2010. IUCN Red List of Threatened Species. Version 2010.1. <http://www.iucnredlist.org/apps/ redlist/details/44495/0> téléchargé le 13 avril 2010

Loiselle, P. et participants à l'atelier CBSG/ANGAP CAMP Faune de Madagascar. 2004. Paretroplus kieneri. In IUCN 2010. IUCN Red List of Threatened Species. Version 2010.1. <http://www.iucnredlist.org/ apps/redlist/details/16235/0> téléchargé le 13 avril 2010

Morschel, J. et Fox, D. 2004. Une méthode de cartographie du risque érosif : application aux collines du Terrefort lauragais. Université de Nice. M@ppemonde 76 (2004.4). <http://mappemonde.mgm.fr/num4/ articles/04404.html> téléchargé le 13 avril 2010

Office National pour I'Environnement 2009. Cahier de Charges Environnementales: Projet de Création de Nouvelle Aire Protégée "Complexe Mahavavy-Kinkony » de Asity Madagascar, District de Mitsinjo, Région Boeny. <http://www.pnae.mg/ee/CCE_CMK_Asity.pdf> téléchargé le 12 mai 2011

Projet ZICOMA 1999. Les Zones d'Importance pour la Conservation des Oiseaux à Madagascar. Antananarivo, Madagascar.

Rabenandrasana, M., Virginie, M. C., Sam, T. S., Randrianarisoa, M. et Zefania, S. 2007. Les zones importantes pour la conservation des oiseaux (ZICO) Mahavavy-Kinkony, un site pilote dans la mise en place d'un site de conservation dans la région ouest de Madagascar: aperçu sur la faune aviaire et les activités de conservation. Ostrish 78: 551-552.

Rabenandrasana, M., Zefania, S., Long, P., Sam, T. S., Virginie, M. C., Randrianarisoa, M., Safford, R. \& Székely, T. 2009. Distribution, habitat and status of 'Endangered' Sakalava Rail of Madagascar. Bird Conservation International 19, 1: 23-32. (doi:10.1017/ S0959270908008058)

Rafalimanana, T. 1989. Résultats de l'Enquête Cadre dans les Principales Pêcheries des Eaux Intérieures de Madagascar 1988/1989. Project reports $n^{\circ} .4$. Rapport non publié remis à la FAO.

Rand, A. L. 1936. The distribution and habits of Madagascar birds. Bulletin of the American Museum of Natural History 72: 134-499.

Roose, E. 1977. Erosion et ruissellement en Afrique de I'Ouest : vingt année de mesures en parcelles expérimentales. ORSTOM, Bondy.
Ségalen, P. 1952. Carte Pédologique de Reconnaissance au 1/200000, Mitsinjo-Mahajanga, Feuille $n^{\circ} 12$, Institut de Recherche Scientifique de Madagascar, ORSTOM, Haut Commissariat de Madagascar et Dépendances.

Wischmeier, W. H. \& Smith, D. 1960. A universal soil-loss estimating equation to guide conservation farm planning. Proceedings of the 7th International Congress of Soil Science 1: 418-425.

\section{MATÉRIEL SUPPLÉMENTAIRE. DISPONIBLE EN LIGNE UNIQUEMENT.}

FIGURE S1. Carte de localisation avec les principales occupations du sol du Complexe Mahavavy-Kinkony, dans la Région de Boeny.

FIGURE S2. Étapes suivies dans le modèle utilisé pour l'élaboration de la carte des zones de faiblesse à l'érosion des bassins versants du lac Kinkony.

FIGURE S3. Délimitation des bassins versants du lac Kinkony réalisée grâce aux rivières et au Modèle Numérique de Terrain. FIGURE S4. Bassins versants du lac Kinkony en fonction de leur faiblesse à l'érosion. (les bassins les plus vulnérables ont le plus grand indice)

FIGURE S5. Extrapolation des valeurs de turbidité sur le plan d'eau du lac Kinkony. (plus la turbidité est importante, plus sa valeur est réduite)

FIGURE S6. Régression linéaire entre la turbidité et la profondeur du lac. 